\title{
Cambios en la Distribución de las Aves: ¿Qué Puerta Hemos Abierto?; los Casos del Chico Piojo (Campylurhynchus rufinucha) y la Paloma Ala Blanca (Zenaida asiatica) en Costa Rica
}

\author{
José Manuel Mora ${ }^{1}$ y Lucía Isabel López ${ }^{2}$
}

\begin{abstract}
Resumen. Los principales factores que afectan la biodiversidad son la pérdida de hábitat, la fragmentación, la sobreexplotación de las especies, la contaminación y la introducción de especies exóticas. A estos se ha agregado una amenaza adicional, el cambio climático global (CCG). Además, las sinergias entre dos o más de estos factores afectarán cada vez más a la biodiversidad. Uno de los efectos previstos, que ya está ocurriendo, es el cambio del ámbito de distribución de las especies. Una de las consecuencias de estos cambios es el reacomodo y la reconformación de las comunidades bióticas y los ensambles de especies. Esto traerá consecuencias bióticas varias de las cuales la más grave es la extinción de especies. Varias especies de aves han aumentado su ámbito de distribución, lo cual podría deberse al aumento de la temperatura debido al CCG y a la pérdida y fragmentación del hábitat. En los últimos años se han reportado la ampliación del ámbito de distribución de varias especies de aves en Costa Rica. Aquí reportamos la ampliación de la distribución del chico piojo (Campylurhynchus rufinucha) y la paloma ala blanca (Zenaida asiatica) desde el bosque seco y el occidente del valle central de Costa Rica a tierras altas de este valle. Además, discutimos acerca del ámbito de distribución de otras especies de aves en Costa Rica. También discutimos los posibles factores y las consecuencias de los cambios de la distribución de las especies en relación a la conservación de la biodiversidad.
\end{abstract}

Palabras clave. Cambio climático global, Cyanocorax affinis, extensión de ámbito, extinción, Psarocolius montezuma.

Changes in the Distribution of Birds: What Door have we opened? The Cases of the Rufous-naped Wren (Campylurhynchus rufinucha) and White-winged Dove (Zenaida asiatica) in Costa Rica

\begin{abstract}
The main factors affecting biodiversity are habitat loss, fragmentation, species overexploitation, pollution and introduction of exotic species. A new threat has to be added, global climate change (GCC). Moreover, synergies between two or more of these factors increasingly affect biodiversity. One of the expected effects, which are already happening, is the change of the species distribution ranges. One consequence of these changes is the relocation and the re-conformation of biotic communities and species assemblages. This will bring several biotic consequences of which species extinctions is the most serious. Several bird species have increased their range, which could be due to the increase in temperature due to the GCC and the loss and fragmentation of the habitat. In recent years several changes in bird species distribution have been reported for Costa Rica. Here, we report the extension of the distribution of Rufous-naped Wren (Campylurhynchus rufinucha) and White-winged Dove (Zenaida asiatica) from the dry forest and western Central Valley of Costa Rica to highland areas of this valley. Furthermore we discuss the range extension of other bird species in Costa Rica. We also discuss the possible factors and consequences of species distribution changes in relation to biodiversity conservation.
\end{abstract}

Keywords. Cyanocorax affinis, distribution range extension, extinction, Global Climate Change, Psarocolius montezuma.

\section{Introducción}

La biodiversidad, esto es la riqueza natural de la vida en todas sus expresiones y niveles, ha sido y es afectada por varios tipos de factores (Wilson 1988). Estos factores pueden ser resumidos en: el deterioro y la destrucción del hábitat, la fragmentación, la sobreexplotación, la introducción de especies exóticas o extranjeras y la contaminación (Ehrlich 1988, Primack 2014). Una amenaza se ha sumado a esta lista en las últimas décadas, el cambio climático (IPCC 2002). El efecto sobre la biodiversidad de cualquiera de las amenazas enlistadas es magnificado por el efecto compuesto (sinergias) que adquiere al accionar en conjunto con otra $\mathrm{u}$ otras de las amenazas (IPCC 2002).

\footnotetext{
${ }^{1}$ Instituto Internacional en Conservación y Manejo de Vida Silvestre (ICOMVIS), Universidad Nacional (UNA), Heredia, Costa Rica. Correo electrónico josemora07@ gmail.com

${ }^{2}$ Consultora independiente. Correo electrónico luciaisa2@gmail.com
}

DOI: $10.5377 /$ ceiba.v52i2.1760 
Los efectos de los factores que afectan la biodiversidad tienen una consecuencia final irremediable que es la pérdida de las especies biológicas (Wilson 1988). Antes de llegar a este punto hay pérdida de la diversidad genética, pérdida de las funciones ecológicas (ej. los muertos vivientes) y la extinción local (Primack 2014). En contraposición están los casos de las especies que, principalmente por ser generalistas, aumentan sus poblaciones y su ámbito de distribución. Pero, en varios casos la explicación de estas ampliaciones de la distribución no es tan clara.

Las aves conforman la segunda clase de vertebrados más diversificada con unas 10425 especies en el mundo (SEO/bird Life 2014). El 13\% de las especies está amenazado de extinción: 213 especies En Peligro Crítico, 419 En Peligro y 741 Vulnerables (SEO/bird Life 2014). De hecho, en la era moderna se han extinguido varias especies por efecto de los factores señalados. Todas las especies de aves conocidas que se han extinguido, desde al año 1600 , han sido por causas humanas (Primack 2014).

Al mismo tiempo, muchas especies de aves han ampliado de forma natural (no introducidas) su ámbito de distribución. Las razones son conocidas o han sido expresadas como hipótesis por varios autores. Aquí reportamos la ampliación del ámbito de distribución en Costa Rica de dos especies de aves características del bosque seco tropical (sensu Holdridge 1967). Basado en estos dos casos de ampliación del ámbito distribucional discutimos las posibles razones que explicarían estas y otras distribuciones de especies de aves en el mismo sitio de referencia. Además, discutimos algunas consecuencias relacionadas a los factores que pueden favorecer la ampliación del ámbito de distribución de algunas especies.

\section{Métodos}

La mayoría de las observaciones fueron en Cinco Esquinas de Carrizal de Alajuela, un pequeño pueblo rural en las faldas del volcán Barva, Costa Rica, a $1700 \mathrm{msnm}$ en el valle Central de Costa Rica $\left(10^{\circ} 06^{\prime}\right.$ $\left.40^{\prime \prime} \mathrm{N}, 84^{\circ} 09^{\prime} 53^{\prime \prime} \mathrm{W}\right)$. Este poblado se encuentra en la vertiente del Pacífico, pero es afectado por los vientos y la humedad del caribe ya que se encuentra cercano al paso de montaña El Desengaño (Savage 2002). La zona de estudio es un área rural con intervención humana desde principios de 1900. Los principales usos del suelo han sido el cultivo de café con sombra y potreros arbolados para ganadería de leche. En las dos o tres últimas décadas, algunas áreas pequeñas de potrero han dado paso a pequeños parches de bosque en regeneración. No obstante, el principal cambio del uso del suelo ha sido la eliminación total del café con sombra para dar lugar a áreas residenciales con jardines y unos huertos caseros pequeños.

Hemos observado aves durante varios años en el área de estudio y en varias otras localidades de Costa Rica. Las observaciones fueron con binoculares, guías de campo y consultas con ornitólogos de Costa Rica. Al mismo tiempo consultamos la literatura especializada y otros medios, particularmente la red de Internet, para conocer casos de especies de aves que han aumentado su distribución en los últimos años.

\section{Resultados}

El chico piojo o salta piñuela (Campylurhynchus rufinucha) es un troglodítido que se distribuye desde Costa Rica hasta México. En Costa Rica se encontraba originalmente en el noroeste del país y la mitad occidental del Valle Central hasta los $1000 \mathrm{msnm}$ (Garrigues y Dean 2007). En los últimos años, el chico piojo ha ampliado su distribución y en el 2004 fue reportado de San Rafael de Heredia a $1110 \mathrm{msnm}$ (Sandoval 2004). Años después, este mismo autor registró a la especie en Concepción de San Rafael de Heredia a 1540 msnm (Sandoval 2013).

En abril de 2014 detectamos al chico piojo en Cinco Esquinas de Carrizal de Alajuela, Costa Rica, a 1694 msnm. El 1 de abril de 2014 los oímos por primera vez en un solar casero. El 7 de abril de 2014 observamos dos chico piojos juntos, forrajeando (insectos) en unos árboles de güitite (Acnistus arborescens) en el mismo sitio de la primera observación. Desde entonces lo hemos observado en varios puntos cercanos al primero. El 24 de abril observamos el primer nido de la especie que estaba siendo construido en un poste del alumbrado público a unos $6 \mathrm{~m}$ de altura.

La paloma aliblanca (Zenaida asiatica) también es una especie característica del bosque seco tropical de Mesoamérica. Esta paloma tuvo la misma 
distribución que el chico piojo en Costa Rica (noroeste del país y mitad occidental del Valle Central, Garrigues y Dean 2007), pero hasta los $500 \mathrm{msnm}$ (Stiles y Skutch 1989). Sin embargo, Garrigues y Dean (2007) señalaron que esta paloma llegaba hasta el este de San José hasta los $1200 \mathrm{msnm}$, lo que Stiles y Skutch (1989) consideraron que obedecía a individuos extraviados. No obstante, esta especie ha venido aumentando su distribución en los últimos años y se ha vuelto muy común incluso en las ciudades. En el campus de la Escuela Agrícola Panamericana en Honduras observamos que la paloma ala blanca se ha convertido en un residente muy común.

El 15 de febrero de 2014 observamos por primera vez una paloma ala blanca en Cinco Esquinas de Carrizal de Alajuela, Costa Rica, donde se ha vuelto abundante. El sitio de observación es casi el mismo donde detectamos al chico piojo. La altitud de 1697 msnm no ha sido incluida dentro del ámbito de distribución conocido para la paloma ala blanca.

Previo a las observaciones señaladas en los párrafos anteriores, detectamos otras especies de aves fuera de su ámbito de distribución (Cuadro 1). Estas distribuciones anómalas o ampliación de ámbito distribucional, no las reportamos debido a varios factores. Cyanocorax affinis fue observada en Cañas Gordas, Coto Brus y Puntarenas, en julio de 2001. Su distribución original era la vertiente del Caribe (Stiles y Skutch 1989). No reportamos ese hallazgo hasta encontrar un nido para describirlo ya que al momento no se conocía (Stiles y Skutch 1989). El año anterior (2000), la especie anidó en el sitio según nos comunicó un vecino del lugar. Posteriormente no tuvimos la oportunidad de visitar más el sitio. El nido y los huevos de Cyanocorax affinis fueron descritos después, así como la ampliación respectiva de distribución de la especie (Martínez et al. 2009).

Otro caso es la oropéndola de Montezuma (Psarocolius montezuma) que se distribuye en Costa Rica en el Caribe, el Valle Central y el noroeste hasta los $1500 \mathrm{msnm}$. En Carrizal de Alajuela estaba ausente hasta febrero de 2014, cuando observamos a diez individuos en una palmera al borde de un cafetal $\left(10^{\circ} 05^{\prime} 43^{\prime \prime} \mathrm{N}, 84^{\circ} 10^{\prime} 15^{\prime \prime} \mathrm{W}\right)$. En junio de 2014, la palmera tenía 18 nidos ocupados por las oropéndolas. Este sitio está a $1490 \mathrm{msnm}$ por lo que no es una adición a la distribución altitudinal de la oropéndola.
Sin embargo, esta es la primera vez que observamos a esta especie en esta localidad. Además, la especie se está estableciendo ya que está anidando en la zona.

El zanate (Quiscalus mexicanus) observado en Cinco Esquinas de Carrizal de Alajuela por primera vez en el 2000, era un hecho conocido que estaba invadiendo abrumadoramente casi todo el país abajo de los 2000 msnm (Garrigues y Dean 2007). A Dives dives lo observamos por primera vez en este sitio en el 2005. No reportamos ese hallazgo debido a que un ornitólogo conocido nos aconsejó obtener primero un espécimen testigo y depositarlo en un museo, lo cual no quisimos hacer en el momento.

\section{Discusión}

La distribución geográfica de las especies ha sido un tema de discusión en ecología por décadas (MacArthur 1972). Una especie se encuentra en un sitio particular solo si éste reúne las condiciones adecuadas para llenar todos los requisitos que ésta demande (Thomas et al. 2004). Dentro de estas condiciones están aspectos relacionados al clima tales como las temperaturas mínimas, máximas y promedio durante el día y a lo largo del año. Además, está la cantidad de lluvia recibida por año y su distribución durante éste (Smith y Smith 2012). A pesar de ser uno de los rompecabezas de más larga discusión en ecología, tenemos poco entendimiento de como la temperatura establece límites geográficos de la distribución de las especies (Sunday et al. 2012).

Varios factores afectan la biodiversidad, varios de ellos, directamente relacionados con las actividades humanas (Ehrlich 1988). El principal factor es la destrucción o alteración del hábitat ya que es uno de los elementos principales para la existencia de un organismo en una localidad. Además de esta afectación clara y directa, la pérdida de hábitat causa cambios ambientales graves que al combinarlos con la pérdida per se afectan más drásticamente a las especies. La fragmentación, la sobreexplotación y la contaminación, junto con la introducción de especies exóticas, disminuyen las posibilidades de supervivencia de la biodiversidad autóctona de un lugar (Primack 2014). A esto hay que agregar un elemento adicional de disturbio a la distribución de los organismos, el cambio climático global (CCG). 
Cuadro 1. Especies de aves observadas por los autores en varios sitios en Costa Rica a alturas (msnm) diferentes a las reportadas por la literatura.

\begin{tabular}{|c|c|c|c|c|c|}
\hline \multirow{2}{*}{ Especie } & \multirow{2}{*}{$\operatorname{msnm} \uparrow$} & \multirow{2}{*}{ Fuente } & \multicolumn{3}{|c|}{ Observación } \\
\hline & & & Fecha & Lugar & $\mathrm{msnm}$ \\
\hline Quiscalus mexicanus & 1500 & Stiles y Skutch 1989 & 2000 & Carrizal & 1700 \\
\hline Cyanocorax affinis & $1300^{\ddagger}$ & Stiles y Skutch 1989 & $07 / 2001$ & Coto Brus & 1075 \\
\hline Dives dives & 500 & Stiles y Skutch 1989 & 2005 & Carrizal & 1700 \\
\hline Zenaida asiática & 1200 & Garrigues y Dean 2007 & $02 / 2014$ & Carrizal & 1694 \\
\hline Campylurhynchus rufinucha & 1540 & Sandoval 2013 & $04 / 2014$ & Carrizal & 1697 \\
\hline Psarocolius montezuma & 1500 & Stiles y Skutch 1989 & $05 / 2014$ & Carrizal & 1500 \\
\hline
\end{tabular}

El CCG amenaza no solo a muchos elementos de la biodiversidad, sino a la especie humana y al planeta y su estabilidad en general (IPCC 2007). Una de las principales consecuencias del CCG es el aumento de la temperatura promedio del planeta (IPCC 2002). En la actualidad hay cambios notorios en varios aspectos de la biodiversidad por el aumento de la temperatura. El riesgo de extinción aumenta cuando el calentamiento global interactúa con otros factores (la modificación del paisaje, las invasiones de especies y la acumulación de dióxido de carbono) para interrumpir las comunidades y las interacciones ecológicas (Pounds y Puschendorf 2004). Varios aspectos no se manifiestan todavía, pero son esperables a mediano y largo plazo, mientras que otros son esperables incluso a corto plazo (IPCC 2002). Se cree que el cambio climático puede amplificar o exacerbar las tensiones existentes en los ecosistemas naturales. Es probable que la resistencia de muchos ecosistemas sea superada en este siglo debido a una combinación sin precedentes de cambio climático y las alteraciones asociadas (inundaciones, sequías, incendios e insectos) y otros problemas ambientales, incluidos el uso de la tierra, la contaminación y la explotación de los recursos (IPCC 2007).

Desde el punto de vista de la conservación de la biodiversidad, un tema de mucha preocupación es el cambio de la distribución de muchas especies de animales y plantas. La redistribución de la vida en la Tierra ha emergido como una de las respuestas de mayor significado biológico al calentamiento global antropogénico (Sunday et al. 2012). Este hecho tendrá consecuencias directas en lo que las áreas silvestres protegidas puedan seguir conteniendo para protección (Hannah et al. 2007). Varias especies ya están experimentado cambios de distribución, relacionados principalmente con la altitud como consecuencia de los cambios en las temperaturas (Hannah et al. 2007).

El calentamiento reciente ha causado cambios en la distribución de las especies y su abundancia, pero el alcance de los efectos no es claro (Pounds et al. 1999). Varias especies de aves se han establecido con un aumento de parejas reproductoras en sitios más altos al que tenían en Monteverde, Costa Rica (Pounds et al. 1999). Aquí se han observado cambios altitudinales en otros grupos taxonómicos que incluyen insectos, anfibios, reptiles, aves y mamíferos (Pounds et al. 2005). En general, existe un desplazamiento hacia los polos y hacia arriba de las montañas en los ámbitos de distribución de las especies de plantas y animales (IPCC 2007). Más del $80 \%$ de las especies que han tenido cambios de desplazamiento están cambiando en la dirección esperada según las limitaciones fisiológicas de las especies (Root et al. 2003).

Siguen vacíos importantes en el conocimiento científico de la forma en que las especies y las comunidades naturales van a responder a las perturbaciones climáticas (Markham y Malcolm 1996), en parte debido a las diferencias regionales en la topografía, microclimas y otros factores (IPCC 2007). Lo que es claro es que los ecosistemas no se mueven en respuesta al cambio climático, lo hacen las 
especies, lo que afectará a las comunidades y las asociaciones de especies. El sinergismo del aumento rápido de la temperatura y otros tipos de estrés, en particular la destrucción del hábitat, podría fácilmente interrumpir la conexión entre las especies y conducir a una reformulación de las comunidades de especies que reflejarían cambios diferenciales en especies y numerosas extirpaciones y posiblemente extinciones (Root et al. 2003). Las especies invasoras, generalistas y ampliamente distribuidas, así como las que se reproducen rápidamente o se dispersan de manera eficiente, es probable que obtengan ventajas competitivas (IPCC 2007). Las especies con mayor probabilidad de ser afectadas negativamente por el cambio climático son los que están en los bordes de su distribución geográfica, las que son raras, tienen poblaciones genéticamente empobrecidas u ocupan nichos de hábitat estrechos y altamente especializados (Markham y Malcolm 1996).

Las tasas de sustitución de especies en algunas comunidades pueden resultar en disturbios ecológicos severos. Una proyección basada en distribuciones de especímenes y algoritmos genéticos desarrolló modelos de nicho ecológico para 1870 especies de la fauna de México para el 2055. Aunque se determinó que las tasas de extinción y reducción drástica de hábitat serían relativamente bajas, se esperaría un alto intercambio de especies, más de $40 \%$ de las especies en algunas comunidades locales (Peterson et al. 2002). En una simulación de escenarios del calentamiento global medio para el 2050, Thomas et al. (2004) estimaron que entre 15 y $37 \%$ de las especies de su muestra de regiones y grupos taxonómicos, de solamente el $20 \%$ de la superficie terrestre del planeta, estarían destinadas a la extinción.

Aparte de lo que pueda suceder con las áreas protegidas y el desplazamiento de su contenido debido al CCG, los cambios en la distribución de algunos animales provocarán otros impactos, algunos de los cuales son predecibles. Varias especies entrarán en contacto con otras con las que no lo tenían. Estos contactos podrán causar efectos de desplazamiento por competencia, hibridación, parasitismo de nidos, promoción de enfermedades infecciosas y otros que podrían ser una amenaza para varias especies, autóctonas o amenazadas de extinción (Pounds et al. 1999). Es conocida la extinción de varias especies o al menos poblaciones de anfibios. En este proceso se ha involucrado al hongo Batrachochytrium dendrobatidis que ha experimentado brotes. La temperatura en las localidades de tierras altas está cambiando a las que son óptimas para el crecimiento de este hongo (Pounds et al. 2006) y de otros parásitos (Pounds et al. 1999).

Los cambios en la distribución de las aves no es solo causada por el aumento de la temperatura, que en algunos casos podrían ser relativamente imperceptibles. Hay otros factores adicionales, dentro de los cuales quizá el principal es el proveer hábitat adecuado a las especies invasoras o la sumatoria de varios aspectos. Esto sucede con las aves que se mueven rápido, comparado con los reptiles y las plantas que se mueven mucho más lento. Esto provoca la desagregación de las comunidades biológicas.

La dispersión del chico piojo y la paloma ala blanca, desde las tierras bajas y medias del bosque seco hasta el valle central y otros sitios de Costa Rica, son la consecuencia de la suma de varios factores. Para el chico piojo el clima podría estar más propicio, pero también el hábitat (Sandoval 2013). Las masas boscosas naturales son filtros para la dispersión de las especies que prefieren hábitats abiertos o perturbados. Esto es así desde los tiempos geológicos y lo ha sido en tiempos históricos. Los bosques tropicales densos de Centro América han sido el filtro de varias especies que de otra manera hubieran migrado desde Norte América hasta Suramérica y viceversa (Gentry 1982, Weir et al. 2009).

La alteración del hábitat ha contribuido a los cambios en la distribución y la abundancia, pero no puede explicar fácilmente los patrones observados a los 1540 msnm en Monteverde, Costa Rica (Pounds et al. 1999). El mosaico de bosques y claros alrededor de la reserva de Monteverde data de la década de 1940 y principios de 1950; no ha habido una deforestación importante en las últimas décadas. Este mosaico, que se encuentra principalmente en las zonas de bosque premontano, debe favorecer la colonización ascendente de las aves de áreas abiertas, que aumentan su abundancia relativa a las aves de bosque donde hay claros. Los patrones observados en dicho sitio de estudio en Monteverde, sin embargo, aplican a ambos grupos de aves (Pounds et al. 1999).

La combinación de la deforestación, aumento de áreas abiertas y el aumento de la temperatura ha 
favorecido a varias especies en Costa Rica. Stiles y Skutch (1989) mencionaron la aparición del tordo cantor (Dives dives) como recientemente registrado en la vertiente del Pacífico y que podía estar invadiendo. Garrigues y Dean (2007) señalaron a dicha especie como de amplia distribución y muy común hasta los 1800 msnm. Esa distribución en Costa Rica la alcanzó el tordo cantor en menos de 20 años. El zanate invadió el Valle Central en 1970 y llegó hasta los 1500 msnm (Stiles y Skutch 1989). Ya en 2007 estaba en casi todo el país hasta los 2000 msnm (Garrigues y Dean 2007). De manera similar, las especies exóticas invasoras pueden ampliar su ámbito de distribución durante el CCG, ya que con frecuencia son oportunistas adaptadas a una gran gama de condiciones (Macdonald 1994).

No tenemos datos de la temperatura ni otros datos meteorológicos en Cinco Esquinas de Carrizal de Alajuela. Las estaciones más cercanas están en el aeropuerto de Alajuela a $15 \mathrm{~km}$ de distancia y a más de $700 \mathrm{~m}$ de diferencia en altitud. Pero, los cambios son claros en la percepción que los habitantes del lugar tienen sobre la disminución de la lluvia, de los vientos, particularmente los temporales, y de temperaturas más altas, principalmente en las noches.

Referente a las aves en el sitio de estudio, es notoria la ausencia de Melozone biarcuatum y Aulocorhynchus prasinus que visitaban comúnmente los cafetales con sombra en esta localidad. En estos cafetales era común observar murciélagos, serpientes e incluso ranas de vidrio (Centrolenidae). Otras aves comunes en el pasado y ausentes ahora son Ptylogonis caudatus, varias eufonías, particularmente Euphonia elelegantisima y el rualdo (Clorophonia calophrys). El rualdo todavía lo observamos en potreros arbolados en sitios cercanos, pero arriba de los $2000 \mathrm{msnm}$. Hace unos 40 años los pajareros venían a este sitio a capturar aves. En la orilla de la carreta (vía al volcán Poás), incluso en nidos, atrapaban eufonías y gallitos (Tiaris olivacea); estas especies no se observan en la zona desde hace varios años.

Los cambios que estamos provocando son imperceptibles para algunos. Sin embargo, son claros para la ciencia e incluso para algunos observadores aficionados o amantes de la naturaleza. Esos cambios podrían llevar a eventos drásticos que afecten aún más a la biodiversidad con un saldo final de mayor pérdida de especies. Por lo tanto, es necesaria la síntesis, no sólo a través de disciplinas, sino entre la teoría y la práctica. Sólo una asociación basada en el manejo adaptativo, en el que los gerentes de campo ayudan a probar y refinar las ideas de manera progresiva, se puede esperar que sea efectiva dadas las incertidumbres de los impactos del CCG en la biodiversidad (Hannah et al. 2002).

Las acciones concretas de protección de la biodiversidad, y particularmente las medidas de mitigación de los efectos del CCG, son una prioridad que no podemos posponer. Acciones en las áreas protegidas, por ejemplo zonas de adaptación al cambio climático, como la incluida en la Reserva Biológica Uyuca en Honduras (Mora y López 2011) son una prioridad. El mantenimiento de la complejidad ecológica y resiliencia de los ecosistemas son los factores más importantes para disminuir los impactos del cambio climático. Las estrategias más eficaces serán las que aumentan la resiliencia a todos los tipos de estrés ambiental y por lo tanto, se debe maximizar la capacidad natural de un ecosistema para adaptarse al cambio. Las inversiones de hoy en la conservación bien pueden ser la mejor defensa contra los efectos del cambio climático (Markham y Malcolm 1996).

\section{Literatura Citada}

Ehrlich, P.R. 1988. The loss of diversity. In E.O. Wilson (Ed.). Biodiversity. National Academy of Sciences, National Academies Press, Washington, D.C. p. 21-22.

Garrigues, R. y R. Dean. 2007. The birds of Costa Rica, a field guide. Comstock Publishing Associates, Ithaca, New York. 387 p.

Gentry, A.H. 1982. Neotropical floristic diversity: Phytogeographical connections between Central and South America, Pleistocene climatic fluctuations or an accident of Andean orogeny? Annals of the Missouri Botanical Garden 69:557-593.

Hannah, L., F. Midgley y D. Millar. 2002. Climate changeintegrated conservation strategies. Global Ecology \& Biogeography 11(6):485-495.

Hannah, L., G. Midgley, S. Andelman, M. Araújo, G. Hughes, E. Martinez-Meyer, R. Pearson y P. Williams. 2007. Protected area needs in a changing climate. Frontiers in Ecology and Environment 5(3):131-138.

Holdridge, L.R. 1967. Ecología Basada en Zonas de Vida. IICA. San José, Costa Rica. 216 p. 
IPCC (Grupo Intergubernamental de Expertos sobre el Cambio Climático). 2002. Cambio climático y biodiversidad. Documento técnico V del IPCC. OMM, WMO, PNUMA y UNEP. Ginebra, Suiza.

IPCC (Intergovernmental Panel on Climate Change). 2007. Mitigation of Climate Change. Contribution of Working Group III to the Fourth Assessment Report. Summary for Policymakers. Disponible en http://www.ipcc.ch/

MacArthur, R.H. 1972. Geographical ecology: patterns in the distribution of species. Harper \& Row Publishers. New York. 269 p.

Markham, A. y J. Malcolm. 1996. Biodiversity and Wildlife: Adaptation to Climate Change. In: J.B. Smith, N. Bhatti, G.V. Menzhulin, R. Benioff, M. Campos, B. Jallow, F. Rijsberman, M.I. Budyko y R.K. Dixon (Eds.). Adapting to Climate Change: An International Assessments and Issues Perspective. (International Conference on Climate Change Adaptation, May 22-25, 1995, St. Petersburg, Russia). Springer. New York, US. p. 384-401

Macdonald, I.A.W. 1994. Global Change and alien invasions: implications for biodiversity and protected area management. In: O.T. Solbrig, H.M. van Emden y P.G.W.J. van Oordt (Eds.). Biodiversity and global change, CAB International, Wallingford, Oxon. p. 199209.

Martínez, A.D. J.E. Sánchez-Pérez y J.R. Zook. 2009. Primer registro del nido y huevos de la urraca pechinegra Cyanocorax affinis, Aves: Corvidae para Costa Rica y notas sobre ampliación de su ámbito de distribución. Brenesia 71/72:79-80.

Mora, J.M. y L.I. López. 2011. El Manejo de la Reserva Biológica Uyuca en el Contexto Nacional y Global del Sistema de Áreas Protegidas. Ceiba 52(1):39-54.

Peterson, A.T., M.A. Ortega-Huerta, J. Bartley, V. SanchezCordero, J. Soberon, R.H. Buddemeier, y D.R.B. Stockwell. 2002. Future projections for Mexican faunas under global climate change scenarios. Nature 416(6881):626-629.

Pounds, J.A. y R. Puschendorf. 2004. Clouded futures. Nature 427(6970):107-109.

Pounds, J.A., M.P.L. Fogden y J.H. Campbell. 1999. Biological response to climate change on a tropical mountain. Nature 398(6728):611-615

Pounds, J.A., M.P.L. Fogden y K.L. Masters. 2005. Responses of natural communities to climate change in a highland tropical forest. In: T.E. Lovejoy y L. Hannah (Eds.). Climate change and biodiversity. Yale University Press, New Haven y London. p. 70-74.

Pounds, J.A., M.R. Bustamante, L.A. Coloma, J.A. Consuegra, M.P. L. Fogden, P.N. Foster, E. La Marca, K.L. Masters, A. Merino-Viteri, R. Puschendorf, S.R.
Ron, G.A. Sánchez-Azofeifa, C.J. Still y B.E. Young. 2006. Widespread amphibian extinctions from epidemic disease driven by global warming. Nature 439(7073):161-167.

Primack, R. 2014. Essentials of conservation biology. Sexta edición. Sinauer Associates. Boston. 603 p.

Root, T.L., J.T. Price, K.R. Hall, S.H. Schneider, C. Rosenzweigk y J.A. Pounds. 2003. Fingerprints of global warming on wild animals and plants. Nature 421(6918):57-60.

Sandoval, L. 2004. Ampliación de ámbito de distribución de dos especies de soterreyes (Troglodytidae: Aves) en Costa Rica. Brenesia 62:99-100.

Sandoval, L. 2013. Cambios en la distribución de aves residentes de Costa Rica. Brenesia 80:59-64.

Savage, J.M. 2002. Amphibians and reptiles of Costa Rica: A herpetofauna between two continents, between two seas. The University of Chicago Press. Chicago, Illinois. $934 \mathrm{p}$.

SEO/Bird Life. 2014. El 13\% de las aves del mundo están amenazadas de extinción. Disponible en http://www.seo.org/2014/07/24/el-13-de-las-aves-delmundo-estan-amenazadas-de-extincion/

Smith, T.M. y R.L. Smith. 2012. Elements of Ecology. 8va ed. Benjamin-Cummings Publishing Company. Boston. $612 \mathrm{p}$.

Stiles, F. G. y A.F. Skutch. 1989. A guide to the birds of Costa Rica. Comstock Publishing Associates. Ithaca, New York. 511 p.

Sunday, J.M., A.E. Bates y N.K. Dulvy. 2012. Thermal tolerance and the global redistribution of animals. Nature Climate Change 2(Sepetember):686-690.

Thomas, C.D., A. Cameron, R.E. Green, M. Bakkenes, L.J. Beaumont, Y.C. Collingham, B.F.N. Erasmus, M.F. de Siqueira, A. Grainger, L. Hannah, L. Hughes, B. Huntley, A.S. van Jaarsveld, G.F. Midgley, L. Miles, M.A. Ortega-Huerta, A.T. Peterson, O.L. Phillips y S.E. Williams. 2004. Extinction risk from climate change. Nature 427(6970):145-148.

Weir, J.T., E. Berminghamb y D. Schlutera. 2009. The Great American Biotic Interchange in birds. PNAS 106(51):21737-21742.

Wilson, E.O. 1988. The current state of biological diversity. In: E.O. Wilson (Ed.). Biodiversity. National Academy of Sciences. National Academies Press, Washington, DC. p 3-18.

Recibido para publicación el 22 de octubre de 2014. Aceptado para publicación el 9 de diciembre de 2014 .. 